\title{
Editorial
}

\section{Economía de la salud: herramientas para la evaluación de programas y políticas públicas}

Núm. 6 (2016), pp. 1-8

Cantarero Prieto, David ${ }^{* 1}$

Oliva Moreno, Juan*2

DOI: $10.5944 /$ reppp.6.2016.16392

\footnotetext{
*1 David Cantarero Prieto. Universidad de Cantabria. Departamento de Economía. Coordinador del número monográfico. E-mail: david.cantarero@unican.es

*2 Juan Oliva Moreno. Universidad de Castilla La Mancha. Departamento de Análisis Económico y Finanzas. Editor Asociado. E-mail: juan.olivamoreno@uclm.es
} 
La Economía de la Salud («Health Economics») aparece como disciplina a partir de la década de 1960 dada la necesidad de analizar tanto el papel que habían alcanzado los sistemas sanitarios públicos como todos aquellos factores que incrementaban su gasto. Así, buena parte del mismo escapa a la acción, al menos directa, de los decisores sanitarios (determinantes de la salud de tipo biológico, medio ambientales, comportamientos y estilos de vida, estructura etaria de la población...), aunque en otros si existe mayores posibilidades de gestión (cartera de servicios, introducción de innovaciones, precios sanitarios,...). De ahí que con el paso del tiempo ha ido cobrando cada vez más relevancia tanto la financiación como la gestión de los recursos empleados en sanidad, dadas sus implicaciones económicas y sociales en términos de bienestar.

Es igualmente importante señalar que, en los principales países desarrollados, la atención sanitaria es de las áreas más reguladas de la economía. Asimismo, la financiación pública de dichos sistemas de salud viene alcanzando más de tres cuartes partes del total, pese a que la mayoría de los bienes y servicios producidos desde la óptica económica tienen la consideración de «bienes privados». Esto es, su consumo es rival (beneficios divisibles) y la posibilidad de exclusión o limitación al acceso es real (tanto a través de un sistema de acceso basado en precios o mediante listas de espera), excepto en los casos particulares de la investigación biomédica o los programas de salud pública. Ello supone que la sanidad es un bien comercializable que puede ser producido privadamente, si bien la Economía de la Salud aporta motivos más que suficientes para justificar la intervención pública en los mercados sanitarios, tanto desde el punto de vista de la equidad como de la eficiencia (corrección de los «fallos de mercado») (Weisbrod, 1961; Arrow, 1963; Grossman, 1972; Fuchs, 1974).

En España, los orígenes de la Economía de la Salud se remontan a finales de la década de 1970 (Bohigas, 2005). No es que anteriormente no hubiera economistas dedicados al análisis del campo sanitario y de la salud. Sin embargo, la confluencia del Encuentro de Economía de la Salud propiciado por el Banco Urquijo y la jornada sobre Economía de la Salud promovida por el Colegio de Economistas de Barcelona, por una parte, permitió traer a España a destacados investigadores del ámbito internacional en calidad de ponentes (Williams, 1980), y por otra, facilitó el contacto de aquellas personas interesadas en esta disciplina. En el año 1980 se celebra la primera Jornada de Economía de la Salud, actividad que se consolida con la creación de la Asociación de Economía de la Salud (AES), en el año 1985, y se mantiene anualmente hasta la celebración de las XXXVI Jornadas en Murcia, en el año 2016. Desde esos primeros años, la internacionalización de la Economía de la Salud ha sido evidente, tanto por la continuada presencia de referentes internacionales en las jornadas y foros científicos organizados en nuestro país como por la creciente salida de profesionales para formarse en los mejores centros mundiales y retornar después o, en algunos casos, desarrollar también su carrera en entidades y universidades extranjeras de prestigio.

El atractivo intelectual de esta disciplina es, sin duda, intenso (Lobo, 1991), su campo de acción amplio y su potencial utilidad social elevada (González López-Varcárcel, 2005). Un repaso a los trabajos premiados en los últimos años por AES nos remite al campo de la evaluación económica de intervenciones sanitarias (Rivero-Arias et al., 2005; Bravo Vergel et al., 2007; González López-Varcárcel y Pinilla, 2008; Mar et al., 2008), al establecimiento de prioridades en el acceso a servicios sanitarios (Sampietro-Colom et al., 2006) y el análisis de equidad en el acceso a dichos servicios (Abásolo et al, 2015), 
al estudio de la competencia entre agentes en mercados con problemas de eficiencia (Olivella y Vera-Hernández, 2007), al estudio de comportamientos de los agentes sanitarios (González, 2005 y González y Macho-Stadler, 2012), al desarrollo de instrumentos para la medición de la salud (Abellán Perpiñán et al., 2011) y la revelación de su valor (Pinto et al., 2009), al análisis del mercado farmacéutico (Puig-Junoy y González LópezValcárcel, 2014) o a las consecuencias sobre la salud de shocks negativos (Cubí-Mollá y Herrero, 2010) y a la importancia, ante estas situaciones, de elementos de carácter institucional (García Gómez et al., 2010). A ello le podríamos sumar el estudio de la relación entre crisis económica y salud, los comportamientos individuales y la importancia de los entornos sociales en el consumo de sustancias adictivas, el análisis de la evolución del gasto sanitario y sus determinantes, los factores que influyen en la introducción de innovaciones en el campo sanitario, el análisis del impacto económico de las enfermedades, el estudio de determinantes sociales de la salud, la investigación sobre variaciones en la práctica médica y un largo etcétera.

Como no se trata de acumular ejemplos sino de sistematizar los campos de análisis que caen bajo el interés de esta disciplina, podemos remitirnos a un esquema conocido como Diagrama de Cajas en Economía de la Salud (Ortún-Rubio et al, 2001; Williams, 1987). Bajo este esquema nos encontraríamos con siete «cajas» o campos de análisis interconectados entre sí. El primero de ellos trataría sobre el análisis de qué es la salud, cómo determinar este concepto (tan escurridizo como el concepto de bienestar), cómo medirlo y valorarlo. En segundo lugar se encontraría el estudio de los determinantes, sanitarios y no sanitarios, de la salud, lo cual nos remite a los conceptos de producción y demanda de salud. En tercer lugar encontraríamos el análisis de la demanda de servicios sanitarios. Dentro del mismo se reunirían factores tan diversos como los determinantes de la demanda de servicios sanitarios, la distinción entre demanda y necesidad, la existencia de barreras de acceso (y, por tanto, el análisis de la equidad en el acceso), la importancia del aseguramiento o las relaciones principal-agente, entre otras. La siguiente «caja» nos remitiría a factores de oferta: funciones de producción sanitaria y eficiencia técnica, costes de producción, coordinación e incentivos en las organizaciones sanitarias, gestión clínica, variaciones en la práctica médica, etc. La quinta caja se centraría en el análisis de los mercados, considerando la interrelación entre aseguradores, proveedores y usuarios, el análisis de los fallos de mercado y la intervención pública para corregirlos, el análisis de los fallos o limitaciones de la intervención pública, los precios monetarios y no monetarios de los servicios sanitarios y las intervenciones y decisiones en salud, los mecanismos de acceso a los servicios no basados en precios (listas de espera) o el análisis del establecimiento de prioridades. A su vez, un siguiente campo de investigación sería la evaluación económica de intervenciones en salud, en sus distintas variantes: análisis de minimización de costes, coste-efectividad, coste-utilidad, coste-beneficio e impacto presupuestario. En la última «caja» nos espera la evaluación, ex ante y ex post, a nivel sistémico. Esto es, incluyendo aspectos de equidad y de eficiencia asignativa, así como el análisis de aspectos concernientes a la planificación, financiación y regulación del sistema sanitarios y de las políticas de salud.

Como en otras disciplinas, o en otras áreas económicas, en las últimas décadas se han producido notables avances en Economía de la Salud, tanto en el campo metodológico, como en el volumen de actividad y generación de resultados. Cabe destacar que, dado su carácter multidisciplinar, con múltiples sinergias y complementariedades con 
las ciencias de la salud, este campo se ha beneficiado tanto de avances en el terreno estadístico-econométrico, enriquecimiento de las bases de datos, métodos experimentales o desarrollo de la economía de las organizaciones, por poner ejemplos muy reconocibles, como por influir en el ámbito de la evaluación económica de programas mediante el desarrollo de herramientas aplicadas al campo de la salud pero exportables a otros ámbitos evaluativos. Del mismo modo, la integración de conceptos procedentes del campo de investigación de servicios de salud en esta disciplina es evidente, pero también lo es su influencia en desarrollos de la propia concepción de la salud, su medición, valoración u orientación ampliadas desde los análisis de oferta de servicios sanitarios hasta el estudio de los determinantes de la salud y las políticas de salud pública.

En este sentido, no es arriesgado afirmar que la Economía de la Salud ha conseguido ya una importante relevancia académica (López Casasnovas y Ortún, 1998; Wagstaff y Culyer, 2012) medida en términos bibliométricos por el índice-h y revistas académicas incluidas en el ISI-JCR Web of Knowledge, así como otros índices de impacto (Health Economics, Journal of Health Economics, Value in Health, Pharmacoeconomics, European Journal of Health Economics, International Journal of Health Care and Managenent, Health Policy, Social Science and Medicine, Economics and Human Biology, Health Economics, Policy \& Law y Applied Health Economics \& Health Policy, entre otras). Siendo clara la relevancia de la disciplina en el campo académico e investigador es más complejo de precisar si los resultados de estas investigaciones siempre se traducen en un impacto social real. En otros países con una cultura evaluativa más desarrollada y donde la rendición de cuentas de los responsables políticos está más asentada socialmente, la influencia de la economía de la salud (y de otras disciplinas) está muy presente, de manera clara y explícita, en los procesos de toma de decisiones. Sin duda, como afirman Ortún y Meneu (2006), la «educación del olfato» de los decisores y la transmisión a la sociedad de la importancia de evaluar nuestras políticas son obligaciones fundamentales de los economistas de la salud ya que de ello depende, en buena medida, el que su cuerpo de conocimiento sea socialmente útil.

En síntesis y echando la vista atrás, mucho se ha avanzado en los últimos años en este campo, (Artells, 1980), generando un importante cuerpo de conocimiento teórico y aplicado. Ya en el presente, esta disciplina sigue intentando dar respuestas a múltiples preguntas de gran trascendencia social, para lo cual debe emplear modelos teóricos rigurosos y los métodos cuantitativos más actuales, al tiempo que progresa en la generación de herramientas específicas para la evaluación de programas y políticas públicas en el campo de la salud (Fuchs, 1974 y 2000; Wagstaff y Culyer, 2012; Pascual y Cantarero, 2013; Zweifel, 2013; Hansen et al., 2015). Los artículos seleccionados en este número monográfico de la Revista de Evaluación de Programas y Políticas Públicas es buena muestra del estado actual de muchos de los aspectos principales de la disciplina en nuestro país descritos en las anteriores líneas.

En el primer trabajo, Trapero-Bertran y Oliva-Moreno (2016) diseccionan el perfil profesional e investigador de las personas que trabajan en el campo de la economía de la salud en España. A través de una encuesta que obtuvo respuesta de 285 personas, el trabajo presenta información sobre las características de estos profesionales, su formación académica, aspectos relacionados con su ocupación, incluyendo ingresos por categoría profesional y tipo de empleo e información sobre la división de su tiempo de trabajo entre diferentes actividades, para pasar a continuación a profundizar sobre el tipo de 
investigación que desarrollan y sus percepciones respecto a aspectos curriculares. Los resultados confirman el carácter multidisciplinar de la economía de la salud en España. Es de especial interés la comparación que realizan los autores en el apartado de discusión entre los resultados españoles y los obtenidos en el Reino Unido y Estados Unidos, en concreto las referidas a ocupación, salarios y empleo del tiempo.

En el segundo de los artículos, Martínez-Pérez et al. (2016) profundizan en un concepto clave en el campo de la economía de la salud y la medición del bienestar: la calidad de vida relacionada con la salud. Aunque trabajos previos habían estimado a escala autonómica la Esperanza de Vida Ajustada por la Calidad (EVAC) en regiones concretas, este artículo proporciona, por primera vez, una estimación para el conjunto de España. Para tal fin, los autores combinan resultados obtenidos de una encuesta representativa de la población adulta española realizada a más de 4.000 personas que incluía el instrumento SF-6D con información de esperanza de vida por sexo y edad proporcionada por el Instituto Nacional de Estadística (INE). Un resultado notable obtenido del trabajo es que las diferencias existentes entre varones y mujeres en términos de esperanza de vida (la esperanza de vida en el momento de nacer se situaba en 84,9 años en el caso de las mujeres y en 78,9 años en varones), al introducir el elemento de calidad de vida relacionada con la salud se reducen considerablemente, de tal manera que entre la EVAC de mujeres y varones en el momento de nacer apenas hay o,6 años de vida ajustados por calidad. Los métodos aplicados y el propio concepto de la EVAC pueden ser de gran utilidad para evaluar los resultados de políticas de salud.

Por su parte, Blázquez et al. (2016) analizan los principales determinantes del gasto sanitario público así como las diferencias en resultados existentes en salud entre las Comunidades de régimen común y las forales. Mediante técnicas econométricas de emparejamiento (matching) se cuantifican esas diferencias para el periodo 2002-2013 obteniéndose como el efecto medio del «tratamiento» de ser considerada región foral (País Vasco y Navarra) incrementa los gastos sanitarios en media en 132,06 euros. Estos resultados apoyan la hipótesis de efectos asimétricos entre distintos tipos de autonomía regional. Así, el que una Comunidad Autónoma sea foral (País Vasco y Navarra), está asociado a unos mayores recursos destinados a sanidad, los cuales tienen su reflejo a su vez en mejores resultados en salud. Los autores señalan que dichas inversiones adicionales en atención sanitaria serían entonces productivas y rentables socialmente y habrían de tomarse en consideración al repensar la próxima reforma del sistema de financiación autonómico en aras a conseguir una mayor equidad.

Cerrando el número, Peña-Longobardo et al (2016) estiman las pérdidas laborales potenciales acaecidas en España en el año 2009. Los autores aplican las técnicas de los estudios de coste de la enfermedad ampliando el objetivo del análisis para dar una fotografía del impacto económico en la parte de pérdida de productividad laboral para el conjunto de enfermedades y lesiones. Esta foto fija se puede añadir a las anteriores estimaciones realizadas por el equipo para los años 2005 y 2007 para tener una panorámica más amplia. Destaca la combinación de varias fuentes de información (INE e Instituto Nacional de la Seguridad Social) para desarrollar un modelo de simulación basado en el enfoque del capital humano que estima las pérdidas asociadas a fallecimientos prematuros, a incapacidad laboral temporal y a incapacidad permanente. Una de las mejoras de este trabajo frente a los anteriores es la estimación de la distribución de las pérdidas por tipo de enfermedad o lesión para cada una de las tres partidas mencionadas. Los 
resultados son contundentes y las pérdidas estimadas totales ascienden a 42.430 millones de euros, una cifra equivalente al 3,93\% del Producto Interior Bruto de España del año 2009. Por tipo de enfermedad o lesión, las enfermedades del sistema osteomuscular y del tejido conjuntivo se sitúan a la cabeza de las pérdidas, seguidas de las causas externas, los trastornos mentales y los tumores. La identificación y la medición de este tipo de costes no sanitarios pueden ser de gran trascendencia en la evaluación de los resultados de políticas y programas de salud, en sentido amplio. Asimismo, varios países incluyen este tipo de costes en la evaluación económica aplicada a nivel micro, en el contexto de financiación pública y negociación del precio y acceso de innovaciones sanitarias.

Confiamos en que a los lectores los artículos seleccionados les resulten de interés, que para aquellos investigadores, profesionales y decisores que desconocían la actividad en el campo de la economía de la salud despierte su curiosidad por esta disciplina y, en suma, que contribuya, siquiera modestamente, a seguir educando nuestro olfato en el terreno de la evaluación de los programas y las políticas públicas.

\section{Agradecimientos}

Deseamos agradecer la comprensiva lectura y las amables sugerencias realizadas a una versión de este editorial de nuestros colegas Joan Josep Artells, Beatriz González LópezVarcárcel, Félix Lobo, Ricard Meneu, Vicente Ortún y Jaume Puig-Junoy. También agradecemos los comentarios del editor del manuscrito. Los errores y omisiones del texto corresponden únicamente a los autores.

\section{Referencias Bibliográficas}

Abásolo, I., Negrín-Hernández, M.A., \& Pinilla, J. (2014). Equity in specialist waiting times by socioeconomic groups: evidence from Spain. The European Journal of Health Economics, 15(3), 323-334.

Abellán Perpiñán, J. M., Sánchez Martínez, F. I., Martínez Pérez, J. E., \& Méndez, I. (2012). Lowering The 'Floor' Of The SF-6D Scoring Algorithm Using A Lottery Equivalent Method. Health economics, 21(11), 1271-1285.

Arrow J. K. (1963). Uncertainty and the welfare economics of medical care. Am Econ Rev.; 53, 941-973.

Artells JJ (1980). La economía de la salud en perspectiva. Cuadernos de Economía 8.21, 5-20.

Blázquez-Fernández, C., Cantarero-Prieto, D., Pascual-Sáez, M. (2016). Políticas públicas de descentralización: ¿̇más gasto y mejores resultados salud? Revista de Evaluación de Programas y Políticas Públicas, 6, 49-65

Bohígas L (2005). 25 años de punto de encuentro. Economía y Salud. Número especial, 52. 
Bravo-Vergel, Y. B., Hawkins, N. S., Claxton, K., Asseburg, C., Palmer, S., Woolacott, N., Bruce I. N., \& Sculpher, M. J. (2007). The cost-effectiveness of etanercept and infliximab for the treatment of patients with psoriatic arthritis. Rheumatology, 46(11), 1729-1735.

Cubí-Mollá, P., \& Herrero, C. (2012). Quality of life lost due to non-fatal road traffic injuries. Health economics, 21(5), 528-550.

Fuchs V (1974). Who Shall Live? Health, Economics, and Social Choice. Basic Books. New York.

Fuchs, V. (2000). The future of Health Economics. Journal of Health Economics, 19, 141-157.

García-Gómez, P. (2011). Institutions, health shocks and labour market outcomes across Europe. Journal of health economics, 3O(1), 200-213.

González, P. (2005). On a policy of transferring public patients to private practice. Health Economics, 14(5), 513-527.

González, P., \& Macho-Stadler, I. (2013). A theoretical approach to dual practice regulations in the health sector. Journal of Health Economics, 32(1), 66-87.

González López-Valcárcel B (2005). ¿Qué hace la economía por nuestra salud? Economía y Salud, Número especial. Mayo, 27-32.

González López-Valcárcel, B. \& Pinilla, J. (2008). The impact of medical technology on health: a longitudinal analysis of ischemic heart disease. Value in Health, 11(1), 88-96.

Grossman, M. (1972). On the Concept of Health Capital and the Demand for Health. Journal of Political Economy, 80(2), 223-255.

Hansen, F., Anell, A., Gerdtham, U., Lyttkens, C.H. (2015). The future of health economics: The potential of behavioral and experimental economics. Nordic Journal of Health Economics, 3(1), 68-86.

Lobo, F. (1991). El atractivo intelectual de la economía de la salud y la asistencia sanitaria. Universidad Carlos III. Madrid: Mimeo.

López I Casasnovas, G., Ortún, V. (1998): Economía y Salud. Fundamentos y políticas. Ediciones Encuentro, Madrid.

Mar, J., Arrospide, A., \& Comas, M. (2010). Budget Impact Analysis of Thrombolysis for Stroke in Spain: A Discrete Event Simulation Model. Value in Health, 13, 69-76.

Martínez-Pérez, J. E., Sanchez-Martínez, F. I, Abellán-Perpiñán, J.M. (2016) Esperanza de Vida Ajustada por la Calidad en España: una aproximación. Revista de Evaluación de Programas y Políticas Públicas, 6, 28-48

Olivella. P., \& Vera-Hernández, M. (2007). Competition among differentiated health plans under adverse selection. $J$ Health Econ. Mar 1, 26(2), 233-50. 
Ortún-Rubio, V., Pinto-Prades. J.L., \& Puig-Junoy, J. (2001). La economía de la salud y su aplicación a la evaluación. Atención Primaria, 27, 62-4.

Ortún-Rubio, V., Meneu de Guillerna, R. (2006). Impacto de la Economía en la política y la gestión sanitaria. Revista Española de Salud Pública, 80, 491-504.

Pascual, M., Cantarero, D. (2013). Understanding health economics: a review of efficiency, equity and inequalities studies. Estudios de Economía Aplicada, 31(2), 1-22.

Peña-Longobardo, L.M, Aranda-Reneo, I., Oliva-Moreno, J., \& Vall-Castello, J. (2016). Evaluación de las pérdidas laborales provocadas enfermedades y lesiones en España en el año 2009. Revista de Evaluación de Programas y Políticas Públicas, $6,66-85$

Pinto Prades, J.L., Loomes, G., \& Brey, R. (2009). Trying to estimate a monetary value for the QALY. Journal of Health Economics, 28, 553-562

Puig-Junoy, J., \& González López-Valcárcel, B. (2014). Launch prices for new pharmaceuticals in the heavily regulated and subsidized Spanish market, 19952007. Health Policy, 116(2), 170-181.

Rivero-Arias, O., Campbell, H., Gray, A., Fairbank, J., Frost, H., \& Wilson-MacDonald, J. (2005). Surgical stabilisation of the spine compared with a programme of intensive rehabilitation for the management of patients with chronic low back pain: cost utility analysis based on a randomised controlled trial. BMJ, 33O(75O2), 1239.

Sampietro-Colom, L., Espallargues, M., Comas, M., Rodríguez, E., Castells, X., \& Pinto, J. L. (2006). Priorización de pacientes en lista de espera para cirugía de cataratas: diferencias en las preferencias entre ciudadanos. Gaceta Sanitaria, 20(5), 342351 .

Trapero-Bertran, M. \& Oliva-Moreno, J. (2016). Profesionales de la economía de la salud: quiénes somos y dónde trabajamos. Revista de Evaluación de Programas y Políticas Públicas, 6, 9-27

Wagstaff, A., Culyer, A.J. (2012). Four decades of health economics through a bibliometric lens. Journal of Health Economics, 31, 406-439

Weisbrod, A.B. (1961): Economics of Public Health. Univ. of Pennsylvania Press.

Williams, A. (1980). Lo que la gente humanitaria debería saber acerca del análisis económico. En: Diálogos sobre Economía de la Salud. Artells JJ, Money G, Williams A editors. Barcelona: Banco Urquijo.

Williams, A. (1987). Health and economics. Oxford: McMillan.

Zweifel, P. (2013). The present state of health economics: a critique and an agenda for the future. European Journal of Health Economics, 14, 569-571. 